\title{
ON MULTIPLE TRANSITIVITY OF PERMUTATION GROUPS
}

\author{
TOSIRO TSUZUKU
}

It is well known that a doubly transitive group $\&$ has an irreducible character $\chi_{1}$ such that $\chi_{1}(R)=\alpha(R)-1$ for any element $R$ of $\mathbb{B}$ and a quadruply transitive group has irreducible characters $\chi_{2}$ and $\chi_{3}$ such that $\chi_{2}(R)=$ $\frac{1}{2} \alpha(R)(\alpha(R)-3)+\beta(R)$ and $\chi_{3}(R)=\frac{1}{2}(\alpha(R)-1)(\alpha(R)-2)-\beta(R)$ where $\alpha(R)$ and $\beta(R)$ are respectively the numbers of one cycles and two cycles contained in $R$. G. Frobenius was led to this fact in the connection with characters of the symmetric groups and he proved the following interesting theorem ${ }^{1}$ : if a permutation group $\&$ of degree $n$ is $t$-ply transitive, then any.irreducible character of the symmetric group of degree $n$ with dimension at most equal to $\frac{t}{2}$ is an irreducible character of $\mathbb{B}$.

In this paper, we shall prove some theorems of a similar type to the above theorem by G. Frobenius which assert multiple transitivity of permutation groups in connection with characters of the symmetric groups. In $\S 1$ we shall sketch some results on the characters of the symmetric groups by G. Frobenius and I. Schur needed in this paper, and in $\S 2$ we prove our theorems. Further, we treat some special cases in $\S 3$.

The auther expresses his gratitudes to Professor T. Nakayama and Professor N. Ito for encouragement and valuable comments.

We use following terminologies and notations. $\Theta^{n}$ designates the symmetric groups of degree $n$ (on $n$ letters $1,2, \ldots, n$ ). $\mathfrak{S}_{1}^{n}$ is the subgroup of $\mathfrak{S}^{n}$ fixing suitable one letter, say 1 , and frequently we identify this group with $\Im^{n-1}$. $\alpha_{1}, \alpha_{2}, \ldots, \alpha_{n}$ are rational integer valued class functions of $\Im^{n}$ such that, for an element $R$ of $\widetilde{S}^{n}, \alpha_{i}(R)$ is the number of cycles of length $i$ contained in $R$, and we say that the type of the element $R$ is $(1)^{\alpha_{1}(R)}(2)^{\alpha_{2}(R)} \cdots(n)^{\alpha_{n}(R)}$. Characters are always ordinary characters and irreducible characters are abso-

1) See [4], \$3.

Received November 30, 1960. 
lutely irreducible characters. For a group $\mathbb{B}$ and its subgroup $\mathscr{S}_{2}, \mathfrak{S}_{2} \chi$ is the restriction to $\$$ of a character $\chi$ of $\&$ and ${ }^{\mathscr{S}} \chi$ is a character of $B$ induced by a character $\chi$ of $\$$. $|\mathbb{B}|$ is the order of a group $\mathbb{B}$.

\section{§1. Frobenius' formula and Schur's recurrence formula ${ }^{2)}$}

If

$$
\boldsymbol{n}=\lambda_{1}+\lambda_{2}+\cdots+\lambda_{n},
$$

is an equation in non negative integers $\lambda_{1}, \lambda_{2}, \ldots, \lambda_{n}$ such that $0 \leqq \lambda_{1} \leqq \lambda_{2} \leqq \cdots$ $\leqq \lambda_{n}$, then the ordered set of non negative integers $\left(\lambda_{1}, \lambda_{2}, \ldots, \lambda_{n}\right)$ (frequently we shall simply write $(\lambda))$ is called a partition of $n$. We may define the order of partitions of $n$ such that $\left(\lambda_{1}, \ldots, \lambda_{n}\right)<\left(\mu_{1}, \ldots, \mu_{n}\right)$ when $\lambda_{n}=\mu_{n}, \lambda_{n-1}=$ $\mu_{n-1}, \ldots, \lambda_{i+1}=\mu_{i+1}$ and $\lambda_{i}>\mu_{i}$ for some $1 \leqq i \leqq n$. Since two elements of $\mathfrak{S}^{n}$ are conjugate if and only if they contain the same numbers of cycles of the same lengths, the number of congugate classes of the symmetric group $\bigodot^{n}$ is equal to the number of partitions of $n$; a partition $(\rho)=\left(\rho_{1}, \ldots, \rho_{n}\right)$ of $n$ corresponds to a conjugate class $\aleph_{(p)}$ consisting of elements of type $\left(\rho_{1}\right)\left(\rho_{2}\right) \ldots$ $\left(\rho_{n}\right)$. So also the number of irreducible characters of $\mathcal{S}^{n}$ is equal to the number of partitions of $\boldsymbol{n}$. All irreducible representations, particularly their characters, are obtained in the rational field. G. Frobenius gave a one to one correspondence between irreducible characters of $\mathcal{\subseteq}^{n}$ and partitions of $n$. If we denote by $\chi^{(\lambda)}$ the character of $\bigodot^{n}$ corresponding to the partition $(\lambda)$ of $n$, then, for any element $R$ of a class $\mathfrak{夭}_{(p)}=\mathfrak{\mho}_{\left(p_{1}, \ldots, p_{n}\right)}$ in $\mathfrak{S}^{n}$,

$$
\chi^{(\lambda)}(R)\left(=\chi_{(\rho)}^{(\lambda)}\right)=\text { the coefficient of } x_{1}^{\alpha_{1}} \cdots x_{n}{ }^{{ }^{k_{n}}} \text { in } F_{(p)},
$$

where $\kappa_{i}=\lambda_{i}+i-1$ and where

$$
\begin{aligned}
F_{(p)} & =\left(x_{1}^{p_{1}}+\cdots+x_{n}^{p_{1}}\right)\left(x_{1}^{p_{2}}+\cdots+x_{n}^{p_{2}}\right) \cdots\left(x_{1}^{p_{n}}+\cdots+x_{n}^{p_{n}}\right) \\
& \times \Delta\left(x_{1}, \ldots, x_{n}\right) \text { where } \Delta\left(x_{1}, \ldots, x_{n}\right)=\prod_{i<j}\left(x_{i}-x_{j}\right),
\end{aligned}
$$

with $n$ variables $x_{1}, \ldots, x_{n}$. This formula is called Frobenius' formula for the characters of $\Im^{n}$. Evidently $F_{(p)}$ is rewritten

$$
\begin{aligned}
\left(x_{1}+\cdots+x_{n}\right)^{\alpha_{1}(R)}\left(x_{1}^{2}+\cdots+x_{n}^{2}\right)^{\alpha_{2}(R)} & \cdots \\
& \left(x_{1}^{n}+\cdots+x_{n}^{n}\right)^{\alpha_{n}(R)} \Delta\left(x_{1}, \ldots, x_{n}\right),
\end{aligned}
$$

for any element $R$ in $\S_{(\rho)}$. We may define the order of irreducible characters

2) See [2], [5] or [6]. 
of $\mathfrak{S}^{n}$ such that $\chi^{(\lambda)}>\chi^{(\mu)}$ when $(\lambda)>(\mu)$. The number $n-\lambda_{n}=\lambda_{1}+\cdots+\lambda_{n-1}$ is called the dimension of $\chi^{(\lambda)}$. For instance, there exists exactly one irreducible character of dimension 0 , i.e. the character $\chi^{(n)},(n)$ meaning $(0, \ldots, 0, n)$; provided $n \geqq 2$ there exists exactly one irreducible character of dimension 1 , $\chi^{(1, n-1)}$; provided $n \geqq 4$ there are two irreducible characters of dimension 2 , $\chi^{(2, n-2)}$ and $\chi^{(1,1, n-2)}$; and provided $n \geqq 6$ there are three irreducible characters of dimension $3, \chi^{(3, n-3)}, \chi^{(1,2, n-3)}$ and $\chi^{(1,1,1, n-2)}$. Here $\chi^{(n)}$ is the unit character $1, \chi^{(1, n-1)}=\alpha_{1}-1, \chi^{(2, n-2)}=\frac{\alpha_{1}\left(\alpha_{1}-3\right)}{2}+\alpha_{2}, \chi^{(1,1, n-2)}=\frac{\left(\alpha_{1}-1\right)\left(\alpha_{1}-2\right)}{2}-\alpha_{2}$ and

$$
\begin{aligned}
\chi^{(3, n-3)} & =\frac{1}{6} \alpha_{1}\left(\alpha_{1}-1\right)\left(\alpha_{1}-5\right)+\left(\alpha_{1}-1\right) \alpha_{2}+\alpha_{3} \\
\chi^{(1,2, n-3)} & =\frac{1}{3} \alpha_{1}\left(\alpha_{1}-2\right)\left(\alpha_{1}-4\right)-\alpha_{3} \\
\chi^{(1,1,1, n-3)} & =\frac{1}{6}\left(\alpha_{1}-1\right)\left(\alpha_{1}-2\right)\left(\alpha_{1}-3\right)-\left(\alpha_{1}-1\right) \alpha_{2}+\alpha_{3},
\end{aligned}
$$

as we readily compute by Frobenius formula.

For the actual calculations of the characters of the symmetric groups we use frequently Schur's well known reccurence formula on the decomposition of an irreducible character of $\mathfrak{S}^{n}$ into those of $\Im_{l}^{n}\left(=\mathfrak{S}^{n-1}\right)^{3)}$. Namely any irreducible character $\chi^{(\lambda)}$ of $\mathfrak{S}^{n}$ is decomposed in $\mathfrak{\subseteq}_{1}^{n}$ as follows; if, for a partition $(\lambda)$ $=\left(\lambda_{1}, \ldots, \lambda_{n}\right)$ of $n,\left(\mu^{(1)}\right), \ldots,\left(\mu^{(r)}\right)$ are all the partitions of $n-1$ appearing in the ordered sets $\left(\lambda_{1}-1, \lambda_{2}, \ldots, \lambda_{n}\right),\left(\lambda_{1}, \lambda_{2}-1, \lambda_{3}, \ldots, \lambda_{n}\right), \ldots,\left(\lambda_{1}, \ldots\right.$, $\left.\lambda_{n-1}, \lambda_{n}-1\right)$, then we have

$$
\Im_{1}^{n} \chi^{(\lambda)}=\chi^{\left(\mu^{(1)}\right)}+\cdots+\chi^{\left(\mu^{(r)}\right)}
$$

\section{§ 2. Main theorems}

By Frobenius' formula it is easy to see that $\chi^{(\lambda)}$ is a polynomial of $\alpha_{1}, \ldots$, $\alpha_{n}$ with rational coefficients. From now on we consider $\alpha_{1}, \ldots, \alpha_{n}$ as variables of weights $1, \ldots, n$ and we define the weight of a monomial $\alpha_{1}^{r_{1}} \cdots \alpha_{n}^{r_{n}}$ as $\sum_{i=1}^{n} i r_{i}$ and the weight of a polynomial $\chi$ of $\alpha_{1}, \ldots, \alpha_{n}$ as the maximum of weights of monomials of $\alpha_{1}, \ldots, \alpha_{n}$ appearing in $\chi$ with non zero coefficients. Firstly we have

3) See [5] or [6]. 
LemMa 1. If the dimension of a character $\chi^{(\lambda)}$ is $r$, then the weight of $\chi^{(\lambda)}$ is at most equal to $r^{4}$.)

Proof. $\chi^{(\lambda)}$ is the coefficient of $x_{1}^{\kappa_{1}} \cdots x_{n}^{\kappa_{n}}$ in (1) where $\kappa_{1}<\cdots<\kappa_{n}, \kappa_{i}=$ $\lambda_{i}+i-1$ and $\lambda_{i}+\cdots+\lambda_{n-1}=r=$ the dimension of $\chi^{(\lambda)}$. The monomial $\chi^{(\lambda)} x_{1}^{\kappa_{1}}$ $\cdots x_{n}^{\kappa n}$ in (1) is obtained as the sum of products of monomials $A_{\mu_{1} \ldots \mu_{n}} x_{1}^{\mu_{1}} \cdots$ $x_{n}^{\mu_{n}}$ in $\left(x_{1}+\cdots+x_{n}\right)^{\alpha_{1}}\left(x_{1}^{2}+\cdots+x_{n}^{2}\right)^{\alpha_{2}} \cdots\left(x_{1}^{n}+\cdots+x_{n}^{n}\right)^{\alpha_{n}}$ and monomials $\pm x_{1}^{\nu_{1}} \cdots x_{n}^{\nu_{n}}$ in $\Delta\left(x_{1} \cdots x_{n}\right)$ such that $\mu_{i}+\nu_{i}=\kappa_{i}$ for $i=1, \ldots, n$. Since $\kappa_{n}$ $=2 n-r-1$ and $\nu_{n} \leqq n-1$, we have $\mu_{n} \geqq n-r$, or, $\sum_{i=1}^{n-1} \mu_{i} \leqq r$. Namely $\chi^{(\lambda)}=$ a sum of $\pm A_{\mu_{1} \ldots \mu_{n}}$ where $\sum_{i=1}^{n-1} \mu_{i} \leqq r$. But it is easy to see that

$$
\begin{aligned}
A_{\mu_{1} \ldots \mu_{n}}= & \sum_{\sum_{i=1}^{n} i \beta_{j}^{(i)}=\mu_{j} \text { for } j=1,2, \cdots, n-1} \prod_{i=1}^{n}\left(\begin{array}{c}
\alpha_{i} \\
\beta_{1}^{(i)}
\end{array}\right)\left(\begin{array}{c}
\alpha_{i}-\beta_{1}^{(i)} \\
\beta_{2}^{(i)}
\end{array}\right) \cdots\left(\begin{array}{c}
\alpha_{i}-\beta_{1}^{(i)}-\cdots \\
\beta_{n}^{(i)}
\end{array}-\beta_{n-1}^{(i)}\right) \\
& \sum_{j=1}^{n} \beta_{j}^{(i)=\alpha_{i} \text { for } i=1,2, \cdots, n}
\end{aligned}
$$

where $\left(\begin{array}{c}\alpha \\ \beta\end{array}\right)=0$ if $\beta<0$ or $\beta>\alpha$. Therefore $A_{\mu_{1} \ldots \mu_{n}}$ with $\sum_{i=1}^{n-1} \mu_{i} \leqq r$ is a polynomial of weight $\leqq r$ in $\alpha_{1}, \ldots, \alpha_{n}$, with rational coefficients. This proves lemma 1 .

1) Professor N. Itô kindly pointed out the following meaning of this lemma; Let \&s be the subgroup $\Xi_{1}, 2, \ldots, r\left(=\mathbb{S}^{n-r}\right)$ of $\mathfrak{S}^{n}$ fixing the $r$ letters $1,2, \ldots, r$ and let $\mathbb{S}_{1}=\mathfrak{S}_{s_{1}}$ $\times \ldots \times \mathscr{S}^{s_{r}} \times \mathfrak{S}^{n-r}$ be a subgroup of the normalisor $\mathfrak{R}(\mathscr{S})$ of $\mathbb{S}$ in $\mathfrak{S}^{n}$ where $\sum_{i=1}^{n} s_{i}=r$ and $0 \leqq s_{1} \leqq s_{2} \leqq \ldots \leqq s_{r}$. Then, by Littlcwood-Richardson's formula, (which is a generalized formula of Schur's one; See D. E. Littlewood [5]) we have

$$
{ }^{\oplus n}\left(\mathbb{B}_{1} 1\right)=\chi\left(s_{1}, s_{2}, \ldots, s_{r}, n-r\right)+\underset{(\rho)<\left(s_{1}, \ldots s_{r}, n-r\right)}{a_{i} \chi(\rho)},
$$

where $a_{i}$ 's are non negative integers. Hence, by inductive consideration it is easy to see that lemma 1 is equivalent to that the weight of ${ }^{{ }^{n}}\left(\mathscr{G}_{1} 1\right)$ is at most equal to $r$. In the set $D$ of elements $\left(1 \underline{i_{1}}\right) \ldots\left(\underline{r}, \underline{i_{r}}\right)$ of $\widehat{S}^{n}$ we define an equivalence relation $\equiv$ such that

$$
\left(1 i_{1}\right) \ldots\left(r i_{r}\right) \equiv\left(1 j_{1}\right) \ldots\left(r j_{r}\right) \text {. }
$$

when $\left\{\underline{i_{1}}, \ldots, \underline{i}_{s_{1}}\right\}=\left\{\underline{j}, \ldots, \underline{j}_{s_{1}}\right\},\left\{\underline{i}_{s_{1}+1}, \ldots, \underline{i}_{s_{1}+s_{2}}\right\}=\left\{j_{s_{1}+1}, \ldots, j_{s_{1}+s_{2}}\right\}, \ldots$ Then it is easy to see that the number of equivalence classes of $D$ is $\frac{n(n-1) \ldots(n-r+1)}{s_{1} ! s_{2} ! \ldots s_{r} !}$ (and this is equal to $\left.\left(\Im^{n} ; \mathfrak{S}_{1}\right)\right)$ and a full representative system $a_{1}, a_{2} \ldots, a_{\left(\Theta^{n}, \Theta_{1}\right)}$ of equivalent classes of $D$ is always a full representative system of the $\Theta^{n}$ by $\uplus_{1}$. For any element $R$ of $\mathbb{B}^{n}$, we have easily

$\left\{{ }^{\Phi^{n}}\left(\Theta_{1} 1\right)\right\}(R)=$ the number of $a_{i}$ such that $a_{i} R a_{i}{ }^{-1} \in \mathbb{G}_{1}$

$$
=\sum_{\sum_{i=1}^{n} i u_{j}^{(i)}=s j} \prod_{i=1}^{n}\left(\begin{array}{c}
\alpha_{i}(R) \\
u_{1}^{(i)}
\end{array}\right)\left(\begin{array}{c}
\alpha_{i}(R)-u_{1}^{(i)} \\
u_{2}^{(i)}
\end{array}\right) \cdots\left(\begin{array}{c}
\alpha_{i}(R)-u_{1}^{(i)}-u_{2}^{(i)}-\ldots-u_{r-1}^{(i)} j \\
u_{r}^{(i)}
\end{array}\right)
$$

where $\left(\begin{array}{l}\alpha \\ \beta\end{array}\right)=0$ if $\alpha<0, \beta<0$ or $\alpha<\beta$. This shows that the weight of $\Theta_{n}\left(\Theta_{1} 1\right)$ is equal to $s_{1}+s_{2}+\ldots+s_{r}=r$. 
Lemma 2. Let $\mathbb{S}$ be a permutation group of degree $n$ and let $r_{1}, \ldots, r_{n}$ be an ordered set of non negative integers.

i) We have always

$$
\sum_{R \in \mathbb{S}}\left(\begin{array}{c}
\alpha_{1}(R) \\
r_{1}
\end{array}\right) \cdots\left(\begin{array}{c}
\alpha_{n}(R) \\
r_{n}
\end{array}\right)=\frac{s|\Theta|}{1^{r_{1}} r_{1} ! 2^{r_{2}} r_{2} ! \cdots n^{r_{n}} r_{n} !},
$$

with a non negative integer s.

ii) (B) is t-ply transitive if and only if

$$
\sum_{R \in \mathscr{S}}\left(\begin{array}{c}
\alpha_{1}(R) \\
r_{1}
\end{array}\right) \cdots\left(\begin{array}{c}
\alpha_{n}(R) \\
r_{n}
\end{array}\right)=\frac{|\&|}{1^{r_{1}} r_{1} ! 2^{r_{2}} r_{2} ! \cdots n^{r_{n}} r_{n} !},
$$

for any ordered set of non negative integers $r_{1}, \ldots, r_{n}$ such that $\sum_{i=1}^{n} i r_{i} \leqq t$, and, if this is the case, for any ordered set of non negative integers $r_{1}, \ldots, r_{n}$ such that $\sum_{i=i}^{n} i r_{i}=t+1$ we have

$$
\sum_{R \in \mathscr{S}}\left(\begin{array}{c}
\alpha_{1}(R) \\
r_{1}
\end{array}\right) \cdots\left(\begin{array}{c}
\alpha_{n}(R) \\
r_{n}
\end{array}\right)=\frac{s_{r, \ldots, r_{n}} ! \& \mid}{1^{r_{1}} r_{1} ! 2^{r_{2}} r_{2} ! \ldots n^{r_{n}} r_{n} !},
$$

with a non negative integer $s_{r_{1} \ldots r_{n}}$ not exceeding the number $s_{0}$ of transitive constituents of $\{t+1, t+\overrightarrow{2}, \ldots, n\}$ by the subgroup $\Theta_{1}, \ldots, t$ of $\$$ fixing $t$ letters $1, \ldots, t$. More precisely

iii) (S) is t-ply transitive if and only if

$$
\sum_{R \in \mathbb{S}}\left(\begin{array}{c}
\alpha_{1}(R) \\
t
\end{array}\right)=\frac{|\mathbb{B}|}{t !}
$$

If $\mathbb{S}$ is exactly $t$-ply transitive, then

$$
\sum_{R \in \mathscr{S}}\left(\begin{array}{c}
\alpha_{1}(R) \\
t+1
\end{array}\right)=\begin{gathered}
s_{0}|\mathfrak{S}| \\
t !
\end{gathered}
$$

Proofs of i) and the "only if" parts of ii), iii) were given in [2], § 1 . We assume $\sum_{R \in \mathscr{S}}\left(\begin{array}{c}\alpha_{1}(R) \\ t\end{array}\right)=\frac{|\&|}{t !}$, i.e. $\sum_{R \in \mathbb{S}} \alpha_{1}(R)\left(\alpha_{1}(R)-1\right) \cdots\left(\alpha_{1}(R)-t+1\right)=|\&|$. Let $\&$ be a permutation group on $n$ letters $\{1,2, \ldots, n\}$. It is easy to see that $\sum_{R \in \mathbb{S}} \alpha_{1}(R)\left(\alpha_{1}(R)-1\right) \cdots\left(\alpha_{1}(R)-t+1\right)$ is equal to the number of all pairs $\left(R, C_{t}\right)$ such that $C_{t}$ is an ordered set with $t$ letters consisting of letters fixed by an element $R$ of $(3$. On the other hand, let $C$ be an ordered subset with $t$ letters of $\{1, \ldots, \underline{n}\}$, say $C=(\underline{1}, \ldots, \underline{t})$, and let $C^{R}$ be the ordered set $\left(1^{R}\right.$, $\left.\ldots, t^{R}\right)$ and let $\mathfrak{S}$ be the subgroup of $(\mathbb{S}$ fixing $t$ letters $1, \ldots, t$. Then, for 


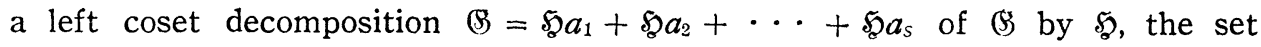
$\left\{\left(a_{i}^{-1} R a_{i}, C^{a_{i}}\right) \mid R \in \mathfrak{S}, i=1, \ldots, s\right\}$ contains $s|\mathfrak{S}|=|\mathfrak{S}|$ pairs, and evidently they are distinct each other, and so any pair exists in this set. Particularly, for any ordered $\operatorname{set}\left(\underline{i_{1}}, \ldots, \underline{i_{t}}\right)$, we have $\left(1,\left(\underline{i_{1}}, \ldots, \underline{i_{t}}\right)\right)=\left(a_{i}^{-1} R a_{i} . C^{a_{i}}\right)$ for some $a_{i}$ and $R$, namely,

$$
\left(1^{a_{i}}, \ldots, \underline{t}^{a_{i}}\right)=\left(\underline{i_{1}}, \ldots, \underline{i_{t}}\right) .
$$

Hence $\mathbb{S}$ is $t$-ply transitive. In this case, the number of conjugate classes of all ordered subsets with $t+1$ letters of $\{1, \ldots, n\}$ by the group $\mathbb{B}$ is evidently equal to $s_{0}$. Let $\widetilde{C}_{1}, \ldots, \widetilde{C}_{s_{0}}$ be a representative system of these conjugate classes and let $\mathscr{S}_{i}$ be the subgroup of $\mathscr{S}$ fixing $t+1$ letters in $\widetilde{C}_{i}$. Then, for a left coset decomposition $\mathbb{B}=\mathscr{S}_{i} a_{1}^{(i)}+\mathscr{S}_{i} a_{1}^{(i)}+\cdots+\mathscr{S}_{i} a_{2 i}^{(i)}$ of $\mathscr{B}$ by $\mathscr{S}^{i}$, the set $D^{(i)}=\left\{\left(a_{j}^{(i)-1} R a_{j}^{(i)}, \widetilde{C}_{i}^{a^{(i,}}\right) \mid R \in \mathscr{S}_{i}, j=1,2, \ldots, u\right\}$ contains $u\left|\mathscr{S}_{i}\right|=|\mathbb{B}|$ pairs, and evidently they are distinct each other. It is easy to see that $D^{(i)} \cap D^{(j)}=\phi$ for $i \neq j$ and any pair $(R, C)$ such that $C$ is an ordered set with $t+1$ letters consisting of letters fixed by an element $R$ of $B$ is contained in some $D^{(i)}$. This proves (4). In similar manner we may prove (3); we omit details.

Now, if $\mathbb{S}$ is the symmetric group $\Theta^{n}$, then, for any integer $t$ such that $0 \leqq t \leqq n$, we have always the relation $\sum_{R \in \mathscr{S}^{n}} \alpha_{1}(R)\left(\alpha_{1}(R)-1\right) \cdots\left(\alpha_{1}(R)-t+1\right)$ $=n !$, because $\Im^{n}$ is $n$-ply transitive. For $t=1$, we have $\sum_{R \in \Im^{n}} \alpha_{1}(R)=n$ ! and, for $t=2, \sum_{R \in \Phi^{n}} \alpha_{1}^{2}(R)=\sum_{R \in \Phi^{n}} \alpha_{1}(R)\left(\alpha_{1}(R)-1\right)+\sum_{R \in \Phi^{n}} \alpha_{1}(R)=2 \cdot n ! . \quad$ In similar manner we can define inductively an arithmetic function $\tau(t)$ such that $\sum_{R \in \widetilde{\sigma}^{n}} \alpha_{1}^{t}(R)$ $=\tau(t) n$ ! for any positive integer $t$. However, for a permutation group $\mathbb{B}$, if $\sum_{R \in \mathscr{S}} \alpha_{1}(R)\left(\alpha_{1}(R)-1\right) \cdots\left(\alpha_{1}(R)-t+1\right)=|\mathbb{S}|$ for all integers $t$ such that $0 \leqq$ $t \leqq m$ then we have also similar relations $\sum_{R \in \mathbb{S}} \alpha_{1}^{t}(R)=\tau(t) \mid(\$)$ for all integers $t$ such that $0 \leqq t \leqq m$. and, conversely, if relations $\sum_{R \in \mathbb{S}} \alpha_{1}^{t}(R)=\tau(t)|\mathbb{S}|$ hold for all integers $t$ such that $0 \leqq t \leqq m$, then we have easily the relation $\sum_{R \in \mathbb{S}} \alpha_{1}(R)\left(\alpha_{1}(R)-1\right)$ $\cdots\left(\alpha_{1}(R)-t+1\right)=|\$|$. Hence, by lemma 2 , we have

LEMмA 3. i) (S) is t-ply transitive if and only if

$$
\sum_{R \in \mathbb{S}} \alpha_{1}^{r}(R)=\tau(r)|\&|
$$

for every integer $r$ such that $0 \leqq r \leqq t$.

ii) (S) is exactly t-ply transitive if and only if 


$$
\sum_{R \in(\mathbb{S})} \alpha_{1}^{r}(R)=\tau(r) \mid(B)
$$

for every integers $r$ such that $0 \leqq r \leqq t$ and

$$
\sum_{R \in \mathbb{S}} \alpha_{1}^{t+1}(R)=\left(\tau(t+1)+s_{0}-1\right)|(\$)|
$$

where $s_{0}$ is the integer in lemma 2 , ii).

Similarly, we can define the arithmetic function $\tau\left(t_{1}, \ldots, t_{n}\right)$ on all ordered sets of $n$ non negative integers $t_{1}, \ldots, t_{n}$ such that

$$
\sum_{R \in \Phi^{n}} \alpha_{1}^{t_{1}}(R) \cdots \alpha_{n}^{t_{n}}(R)=\tau\left(t_{1}, \ldots, t_{n}\right) n !
$$

and we have

Lemma $3^{\prime}$. (S) is t-ply transitive if and only if

$$
\sum_{R \in \mathbb{S}^{S}} \alpha_{1}^{t_{1}}(R) \cdots \alpha_{n}^{t_{n}}(R)=\tau\left(t_{1}, \ldots, t_{n}\right)|\&|
$$

for every ordered set $\left(t_{1}, \ldots, t_{n}\right)$ such that $\sum_{i=1}^{n} i t_{i} \leqq t$, and further if $\&$ is exactly $t$-ply transitive, then we have

$$
\sum_{R \in \mathscr{S}} \alpha_{1}^{t_{1}}(R) \ldots \alpha_{n}^{t_{n}}(R) \supsetneqq \tau\left(t_{1}, \ldots, t_{n}\right)|\mathbb{S}|,
$$

for every ordered set $\left(t_{1}, \ldots, t_{n}\right)$ such that $\sum_{i=1}^{n} i t_{i}=t+1$. and $s_{t_{1}} \ldots t_{n}$ (of lemma $2, i i)) \neq 0$.

$\alpha_{1}(R)$ is a character of $\stackrel{\bigodot}{ }^{n}$, since $\alpha_{1}(R)-1$ is a character, and so, for any non negative integer $r, \alpha_{1}^{r}(R)$ is a character of $\Im^{n}$. Class functions $\prod_{i=1}^{n} \alpha_{i}^{r_{i}}(R)$, for $r_{i} \geqq 0$, are not always characters, but we have

LemMA 4. A class function $\prod_{i=1}^{n} \alpha_{i}^{\mu_{i}}$ of $\Im^{n}$ of a weight $r \leqq \frac{n}{2}$ (i.e. $\sum_{i=1}^{n} i \mu_{i}=r$, $\left.\mu_{i} \geqq 0\right)$ is a linear combination of irreducible characters of dimensions $\leqq r$ with rational coefficients.

Proof. For $r=1$, this is obious. We assume that for any integer $s \leqq r-1$ our assertion is correct. Let $\chi_{:}, \ldots, \chi_{p(r)}$ be all irreducible characters of dimension $r$. By the difinition of the dimension, $\rho(r)$ is equal to the number of partitions of $r$. On the other hand, the number of monomials of $\alpha_{1}, \ldots, \alpha_{n}$ of aweight $r$ is also equal to the number of partitions of $r$ and we denote these $\rho(\boldsymbol{r})$ monomials by $M_{1}, M_{2}, \ldots, M_{\rho(r)}$. By Lemma 1 , 


$$
\begin{aligned}
& \%_{1}=a_{1},{ }_{1} M_{1}+\cdots+a_{1},{ }_{p(r)} M_{\rho(r)}+f_{1} \\
& \chi_{2}=a_{2},{ }_{1} M_{1}+\cdots+a_{2},{ }_{\rho}(r) M_{\rho}(r)+f_{2} \\
& \chi_{p(r)}=a_{p(r),}{ }_{1} M_{1}+\cdots+a_{p(r), p(r)} M_{p(r)}+f_{p(r)},
\end{aligned}
$$

where $f_{1}, f_{2}, \ldots, f_{\rho}(r)$ are polynomials of $\alpha_{1}, \ldots, \alpha_{n}$ of weights $<r$ with rational integral coefficients and, by the inductive assumption, they are linear combinations of irreducible characters of dimension $\leqq r-1$ with rational coefficients. Since $\chi_{1}, \ldots, \chi_{p(r)}$ are of dimension $r, \chi_{i}-f_{i}, i=1, \ldots, \rho(r)$, are independent class functions of $\Subset^{n}$. Hence simultaneous equations

$$
\chi_{i}-f_{i}=a_{i},{ }_{1} M_{1}+\cdots+a_{i},{ }_{\rho}(r) M_{\rho(r)}, i=1, \ldots, \rho(r)
$$

have unique solutions $M_{i}, i=1, \ldots, \rho(r)$, and they are linear combinations of irreducible characters of dimensions $\leqq r$ with rational coefficients.

LemMa 5. Let $\&$ be a permutation group of degree $n$ and let $r$ be a positive integer such that $r<\frac{n}{2}$. If for at least one character $\chi^{(\lambda)}$ with dimension $r$ of $\mathbb{S}^{n}$ the character $\mathbb{S} \chi^{(\lambda)}$ of $\mathbb{S}$ is irreducible, then $\mathbb{B}$ is transitive.

Proof. If we assume that $B$ is intransitive, then $B$ is contained in a subgroup $\mathfrak{S}^{n_{1}} \times \bigodot^{n_{2}}$ of $\Im^{n}$ for some positive integers $n_{1}, n_{2}$ such that $n=n_{1}+n_{2}$, where $\subseteq^{n_{1}}$ is the symmetric subgroup of $\Im^{n}$ on $n_{1}$ letters $\underline{1}, 2, \ldots, n_{1}$ fixing the other $n-n_{1}$ letters $n_{\mathrm{i}}+1, \ldots, \underline{n}$ and $\mathcal{S}^{n_{2}}$ is the symmetric subgroup of $\Xi^{n}$ on $n_{1}+1, \ldots, \underline{n}$ fixing $1, \ldots, n_{1}$. Hence, to prove our lemma, it is

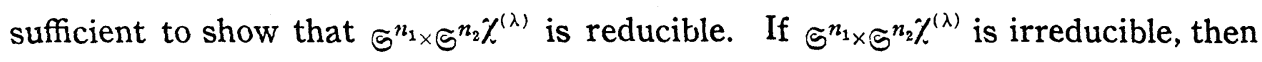
this is a Kronecker product of irreducible characters of $\varsigma^{n_{1}}$ and $\varsigma^{n_{2}}$, and so each of $\Im^{n_{1}} \%^{(\lambda)}$ and $\Im^{n_{2}} \%^{(\lambda)}$ is a multiple of one irreducible character, of $\Im^{n_{1}}$ and $\Xi^{n_{2}}$ respectively. But this is impossible by our assumption $0<r<\frac{n}{2}$ and Schur's recurrence formula. In fact if $n_{1} \leqq n_{2}$, then $n_{2}$ is greater than the dimension of $\chi^{(\lambda)}$ and so we have $\mathbb{S}^{n_{1} \varkappa^{(\lambda)}}=\mathcal{X}^{\left(n_{1}\right)}+\mathcal{K}^{\left(1, n_{1}-1\right)}+\cdots$ by Schur's recurrence formula.

Lemma 6. Let $\mathbb{B}$ be a subgroup of $\mathfrak{S}^{n}$ and let $r$ be a positive integer such that $r<\frac{n}{2}$. If every character $\& \%^{(\lambda)}$ of $\&$ such that the dimension of $\varkappa^{(\lambda)}$ is $r$ is irreducible, then every character $\mathscr{S}_{1} \widehat{\chi}^{(\mu)}$ of $\mathfrak{S}_{1}=\mathscr{S} \cap \mathfrak{S}_{1}^{n}$ such that the dimension of $\tilde{\chi}^{(\mu)}$ is $r-1$ (we regard $\mathbb{S}_{1}$ as a subgroup of $\mathfrak{\subseteq}^{n-1}$ identified with $\Im_{1}^{n}$ and $\tilde{\psi}^{(\mu)}$ is an irreducible character of $\left.\mathfrak{\Im}^{n-1}\right)$ is irreducible.

Proof. By lemma $5, \mathbb{B}$ is a transitive group. Hence a full representative 
system of the left coset decomposition of $\mathbb{B}$ by $\mathscr{B}_{1}$ is also a full representative system of that of $\Im^{n}$ by $\mathbb{S}_{1}^{n}$. So, by the definition of induced characters,

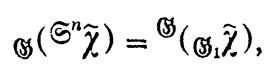

for any character $\tilde{\chi}$ of $\mathfrak{S}_{1}^{n}$. By Schur's recurrence formula, for an irreducible character $\varkappa^{(\lambda)}$ of $\mathfrak{S}^{n} \mathfrak{S}_{1}^{n} \mathcal{\varkappa}^{(\lambda)}$ is the sum of distinct irreducible characters $\tilde{\chi}^{(\mu)}$ of $\Im_{1}^{n}$ and if the dimension of $\chi^{(\lambda)}$ is $r$, then the dimension of $\tilde{\chi}^{(\mu)}$ is either $r$ or $r-1$ for any irreducible character $\tilde{\chi}^{(\mu)}$ appearing in $\varsigma_{1}^{n} \chi^{(\lambda)}$.

Now we assume that, for some irreducible character $\tilde{\chi}_{0}$ of $\Im_{1}^{n}$ of dimension $r-1, \Im_{1} \tilde{\chi}_{0}$ is reducible, namely

$$
\mathbb{G}_{1} \tilde{\chi}_{0}=\psi_{0,1}+\%_{0,2}+\cdots \chi_{0, s}
$$

where $s \geqq 2$ and $\psi_{0, i}, i=1, \ldots, s$, are irreducible characters of $\mathbb{S}_{1}$. Let $\chi_{1}$ be any irreducible character of $\mathbb{S}^{n}$ of dimension $r$ appearing in $\Im^{n} \tilde{\chi}_{0}$. Then

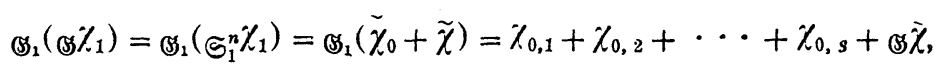

where $\hat{\chi}$ is a character of $\mathfrak{S}_{1}^{n}$, and by the reciprocity theorem on group characters and by the irreduciblity of $s \%$ we have

$$
{ }^{\mathscr{S}}\left(\mathbb{S}_{1} \hat{\chi}_{0}\right)={ }^{\mathfrak{G}}\left(\chi_{0,1}+\chi_{0,2}+\cdots+\chi_{0, s}\right)=s \times \operatorname{si} \chi_{1}+\cdots,
$$

where $s \geqq 2$.

On the other hand, for any irreducible character $\widetilde{\psi}_{0}$ of $\varrho_{1}^{n}$ of dimension $r-1$ we have

$$
\mathbb{S}^{n} \tilde{\chi}_{0}=\chi_{1}+\cdots+\chi_{t}+\chi_{t+1},
$$

where $\psi_{1}, \ldots, \psi_{t}$ are irreducible characters of $\mathbb{S}^{n}$ of dimension $r$ and $\psi_{t+1}$ is an irreducible character of $\mathcal{S}^{n}$ of dimension $r-1$, and so we have

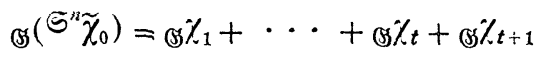

where $\mathscr{s} \%$ is an irreducible character of $\mathbb{B}$ for $i=1, \ldots, t$. $\mathscr{s} \% t+1$ may be a reducible character, but at least one character $s \% i$ does not appear as an irreducible component of $s \chi_{t+1}$. To see this, it is sufficient to show that the degree of ${ }_{\mathscr{S}}\left(\mathcal{S}^{n} \tilde{\chi}_{0}\right)$ is larger than twice of the degree of $\leftrightarrow \psi_{t+1}$. Let $\tilde{\chi}_{0}=\chi^{(\lambda)}$ where $(\lambda)$ $=\left(\lambda_{1}, \ldots, \lambda_{n-1}\right)$ is a partition of $n-1$ and so $\chi_{t+1}=\chi^{(\mu)}$ where $(\mu)=\left(0, \lambda_{1}, \lambda_{2}\right.$, $\left.\ldots, \lambda_{n-2}, \lambda_{n-1}+1\right)$ is a partition of $n$. By Frobenius's formula. 
the degree of $\mathscr{S S}\left(\mathcal{S}^{n} \widetilde{\gamma}_{0}\right)=n \times \frac{(n-1) ! \Delta\left(\lambda_{1}, \lambda_{2}+1, \ldots, \lambda_{n-1}+n-2\right)}{\lambda_{1} !\left(\lambda_{2}+1\right) ! \cdots\left(\lambda_{n-1}+n-2\right) !}$

the degree of $\psi_{t+1}=\frac{n ! \Delta\left(\lambda_{1}, \lambda_{2}+1, \ldots, \lambda_{n-2}+n-3, \lambda_{n-1}+n-1\right)}{\lambda_{1} !\left(\lambda_{2}+1\right) ! \cdots\left(\lambda_{n-2}+n-3\right) !\left(\lambda_{n-1}+n-1\right) !}$,

and so we have

the degree of os $\left.^{\left(S_{0}\right.}\right) \quad \Delta\left(\lambda_{1}, \lambda_{2}+1, \ldots, \lambda_{n-1}+n-2\right)$

$2 \times$ the degree of $\psi_{t+1}=\overline{\Delta\left(\lambda_{1}, \lambda_{2}+1, \ldots, \lambda_{n-2}+n-3, \lambda_{n-1}+n-1\right)}$

$$
\begin{aligned}
& \times \frac{\lambda_{n-1}+n-1}{2} \\
= & \frac{\lambda_{n-1}+n-1}{2} \prod_{i=1}^{n-2}\left(1-\frac{1}{\lambda_{n-1}-\lambda_{i}+n-i}\right) \\
= & \frac{2 n-r-1}{2} \prod_{i=1}^{n-2}\left(1-\frac{1}{\lambda_{n-1}-\lambda_{i}+n-i}\right),
\end{aligned}
$$

and, since $r<\frac{n}{2}$ and $\lambda_{n-1} \geqq \lambda_{i}$, this is

$$
>\frac{\frac{3}{2} n}{2} \prod_{i=1}^{n-2}\left(1-\frac{1}{n-i+1}\right)=\frac{3 n}{4} \times \frac{2}{n}>1 .
$$

Namely, we have proved that, for any irreducible character $\tilde{\chi}_{0}$ of dimension $r-1$ of $\Theta_{1}^{n}$, there is always an irreducible character $\psi_{1}$ of dimension $r$ of $\mathfrak{S}^{n}$ such that $\& \psi_{1}$ appears exactly once in $\mathbb{S}_{(}\left(\mathbb{S}^{n} \tilde{\chi}_{0}\right)={ }^{\mathbb{S}}\left(\mathbb{S}_{1} \widetilde{\chi}_{0}\right)$. If we assume that $\Im_{1} \widetilde{\chi}_{0}$ is reducible for some irreducible character $\widetilde{\chi}_{0}$ of dimension $r-1$ of $\Im_{1}^{n}$, this fact contradicts with (5).

Now we can prove our theorems:

Theorem 1. (G. Frobenius [2]) Let $\&$ be a permutation group. If $\mathbb{B}$ is $2 r$-ply transitive, then for any irreducible character $\%$ of dimension $\leqq r$ of $\mathfrak{S}^{n}$, the character $\mathbb{S} \%$ of $\mathbb{B}$ is irreducible.

Proof. By lemma 1, we can write $\%=\sum_{i} a_{i} M_{i}$ where $M_{i}$ 's are monomials of $\alpha_{1}, \ldots, \alpha_{n}$ with weights $\leqq r$. Hence we have $\sum_{R \in \mathscr{S}}\left(\AA^{2}(R)=\sum_{i, j} a_{i} a_{j} \sum_{R \in \mathscr{S}}\left(M_{i} M_{j}\right)(R)\right.$ and, since weights of $M_{i} M_{j} \leqq 2 r$, by lemma $3^{\prime}$

$$
\sum_{R \in \mathbb{S}}\left(M_{i} M_{j}\right)(R)=\tau\left(M_{i} M_{j}\right)|\&|,
$$

where $\tau\left(M_{i} M_{j}\right)=\tau\left(t_{1}, \ldots, t_{n}\right)$ when $M_{i} M_{j}=\alpha_{1}^{t_{1}} \ldots \alpha_{n}^{t_{n}}$. So we have

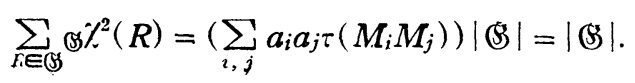


Hence $s \%$ is irreducible.

Theorem 2. Let $\&$ be a permutation group of degree $n$ and let $r<\frac{n}{2}$. If for every character $\chi$ of dimension $r$ of $\mathbb{S}^{n}$ the character $\& \%$ of $\mathbb{\&}$ is irreducible, then \& is $2 r$-ply transitive.

Proof. For $r=1$, there is only one character $\chi_{0}$ of dimension $1 ; \psi_{0}=\alpha_{1}-1$. If $\& \chi_{0}$ is irreducible, then $\sum_{R \in \mathbb{S}}\left(\alpha_{1}(R)-1\right)=0$ and $\sum_{R \in \mathbb{S}}\left(\alpha_{1}(R)-1\right)^{2}=|\mathbb{B}|$, hence we have

$$
\sum_{R \in \mathbb{S}} \alpha_{1}(R)=|\mathbb{B}| \text { and } \sum_{R \in \mathbb{S}} \alpha_{1}^{2}(R)=2|\mathbb{\&}| .
$$

On the other hand, since, by the definition of the function $\tau, \tau(1)=1$, and $\tau(2)$ $=2, \mathbb{B}$ is doubly transitive by lemma 3 . We shall proceed by induction on $r$. Let $\mathbb{S}_{1}$ be the subgroup of $\mathbb{S}$ fixing one letter. Then, by lemma 6 and inductive assumption, $\mathbb{B}_{1}$ is $(2 r-2)$-ply transitive and so $\mathbb{B}$ is $(2 r-1)$-ply transitive. Hence, by Theorem 1 and our assumption, every character of dimension $\leqq r$ is

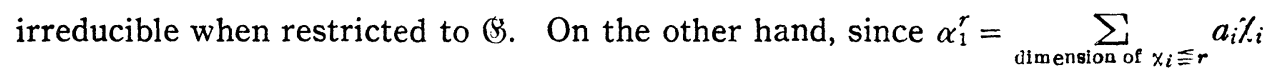
by lemma 4 , we have

$$
\sum_{R \in \mathscr{S}} \alpha_{1}^{2 r}(R)=\sum_{i, j} a_{i} a_{j} \sum_{R \in \mathscr{S}} \chi_{i}(R) \chi_{j}(R)=\left(\sum_{i} a_{1}^{2}\right)|\mathbb{S}| \text {. }
$$

From the relation $\sum_{R \in \mathbb{S}^{n}} \alpha_{1}^{2 r}(R)=\tau(2 r) n !$ we have $\sum_{i} a_{i}^{2}=\tau(2 r)$ and so

$$
\sum_{R \in \mathbb{S}} \alpha_{1}^{2 r}(R)=\tau(2 r)|\mathfrak{B}| \text {. }
$$

Hence $\mathbb{B}$ is $2 \boldsymbol{r}$-ply transitive by lemma 3 .

\section{§. Special cases}

By Theorem 1 and 2 we know that the irreducibility of the restriction of the (unique) character of the dimension 1 is equivalent to the double transitivity of the group and the irreducibility of the restrictions of two (i.e. all) characters of dimension 2 is equivalent to the quadruple transitivity of the group. In this section we study what we can say by the irreducibility of the restriction of one of the characters of dimensions 2 and 3.

Theorem 3. Let \& be a permutation group of degree $n \geqq 5$. If either $\mathfrak{G} \%^{(2, n-2)}$ or $\mathfrak{G S}^{(1,1, n-2)}$ is irreducible, then $\mathbb{B}$ is triply transitive.

Proof. Case I. Let $\leftrightarrow \%^{(2, n-2)}$ be irreducible. If $\leftrightarrow \%^{(1,1, n-2)}$ is irreducible, it 
is correct by Theorem 2 and so we can assume that $(\$) \%^{(1,1, n-2)}$ is reducible. \& is transitive by lemma 5 .

i) $\mathbb{B}$ is doubly transitive. Let us assume the contrary i.e. that $\mathbb{B}$ is not doubly transitive. Since $s \%^{(2, n-2)}$ is irreducible, we have

$$
\sum_{R \in \mathbb{S}}\left(\frac{\alpha_{1}(R)\left(\alpha_{1}(R)-3\right)}{2}+\alpha_{2}(R)\right)=0
$$

and so, by lemma 3, i)

$$
\frac{1}{2} \sum_{R \in \mathbb{S}} \alpha_{1}^{2}(R)+\sum_{R \in \mathbb{S}} \alpha_{2}(R)=\frac{3}{2}|\mathbb{S}| \text {. }
$$

Since $\mathbb{B}$ is not doubly transitive, $\sum_{R \in \mathscr{S}} \alpha_{1}^{2}(R)>2|\mathbb{B}|$ by lemma 3 , ii). Therefore, by (6), we have $\sum_{R \in \mathscr{S}} \alpha_{1}^{2}(R)=3|\&|$ and $\sum_{R \in \mathbb{S}} \alpha_{2}(R)=0$ i.e. ${ }_{\mathbb{S}} \alpha_{2}=0$ and so $|\&|$ and $n$ are odd numbers and since $|\mathcal{B}|$ is divisible by $\frac{n(n-3)}{2}$ (the degree of $\left.\chi^{(2, n-1)}\right)$, $n \equiv 1 \bmod 4$. Hence

$$
\begin{aligned}
& \text { (s) } \mathcal{L}^{(2, n-2)}=\frac{1}{2}-\alpha_{1}\left(\alpha_{1}-3\right)
\end{aligned}
$$

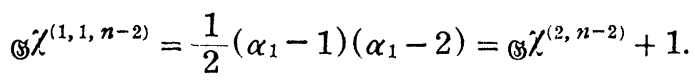

On the other hand, for $\mathbb{S}^{n-1}\left(=\mathbb{S}_{1}^{n} \subset \mathfrak{S}^{n}\right)$ and its subgroup $\mathbb{S}_{1}=\mathbb{S} \cap \mathfrak{S}^{n-1}$, we have similarly

$$
\mathscr{F}_{1} \chi^{(1,1, n-3)}=\mathbb{S}_{1} \chi^{(2, n-3)}+1
$$

By the reciprocity law, we have

$$
\begin{aligned}
& \Im^{n}\left(\mathscr{S}^{(2, n-2)}\right)=\chi^{(1,1, n-2)}+\chi^{(2, n-2)}+\mathrm{a} \text { sum of irreducible characters of } \\
& \mathfrak{S}^{n} \text { whose dimensions }>2 \text {. }
\end{aligned}
$$

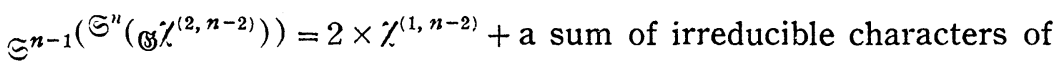

$$
\begin{aligned}
& \varsigma^{n-1} \text { whose dimensions } \geqq 2 \text {. }
\end{aligned}
$$

Therefore $\mathfrak{G}_{1} \%^{(1, n-2)}$ is a sum of exactly two irreducible characters, by the reciprocity law, and, since $\mathscr{S}_{1}$ is not transitive, $\mathscr{G}_{1} \mathcal{H}^{(1, n-2)}$ contains the unit character by lemma 3 , ii). Hence there is an irredacible character $\tilde{\psi}$ of $\left(\mathscr{S}_{1}\right.$ such that the degree of $\tilde{\chi}$ is $n-3$ and so $\left|\mathbb{S}_{1}\right|$ must be divisible by $n-3$. This is impossible, because $n\left|\mathfrak{B}_{1}\right|$ is odd.

ii) $(\mathbb{B}$ is triply transitive. Assume that $\mathbb{B}$ is not triply transitive. Since 
(S) is doubly transitive, of $Z_{1}^{(1, n-1)}$ is irreducible. If $\mathscr{O S}_{1} \chi^{(1, n-2)}$ is irreducible then $\mathbb{S}_{1}$ is doubly transitive and so $\mathbb{B}$ is triply transitive. Therefore $\mathscr{G}_{1} \mathcal{Z}^{(1, n-2)}$ is reducible and, consequently, $\Xi_{n-1}\left(\Xi^{n}\left((s) \chi^{(2, n-2)}\right)\right)$ contains $\chi^{(1, n-2)}$ at least twice, by reciprocity law. On the other hand, since $\leftrightarrow \%^{(2, n-2)}$ is irreducible, $\Im^{n}\left(\leftrightarrow \%^{(2, n-2)}\right)$ contains $\varkappa^{(2, n-2)}$ exactly once. Therefore $\widetilde{S}^{n}\left((\mathscr{S})^{(2, n-2)}\right)$ contains $\%^{(1,1, n-2)}$ and so we have

$$
(S) \%^{(1,1, n-2)}=\left(s_{5} \%^{(2, n-2)}+\left(1-2 \alpha_{2}\right),\right.
$$

and, since the degree of $\chi^{(1,1, n-2)}=$ the degree of $\chi^{(2, n-2)}+1$, the degree of the character $\left(1-2 \alpha_{2}\right)$ is 1 and so the character $\left(1-2 \alpha_{2}\right)$ is irreducible. Hence, since $\mathfrak{s} \%^{(1, n-1)}$ is irreducible and is not equal to $\left(1-2 \alpha_{2}\right)$ nor to $\left.\mathfrak{s}\right)^{(2, n-2)}$, we have

$$
\begin{aligned}
& \sum_{R \in(\mathbb{S})}\left(\frac{\alpha_{1}(R)\left(\alpha_{1}(R)-3\right)}{2}+\alpha_{2}(R)\right)\left(\alpha_{1}(R)-1\right)=0 \\
& \sum_{R \in \mathbb{S}}\left(\frac{\left.\alpha_{1}(R)-1\right)\left(\alpha_{1}(R)-2\right)}{2}-\alpha_{2}(R)\right)\left(\alpha_{1}(R)-1\right)=0
\end{aligned}
$$

and so we have $\sum_{R \in \mathscr{S}} \alpha_{1}^{3}(R)=5|\mathfrak{G}|=\tau(3)|\mathbb{S}|$. Hence $\mathbb{S}$ is triply transitive by lemma 3, i).

Case II. Let of $7^{(1,1, n-2)}$ be irreducible. In this case, by Theorem 2 , it is sufficient to show that $\sigma_{1} \chi^{(1, n-2)}$ is irreducible. We assume that $\tilde{\sigma}_{1} \chi^{(1, n-2)}$ is

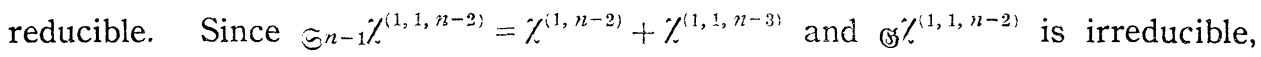
${ }^{S}\left(\mathscr{S}_{1} \mathcal{Z}^{(1, n-2)}\right)$ contains $\mathscr{S} \%^{(1,1, n-2)}$ at least twice. On the other hand, since the

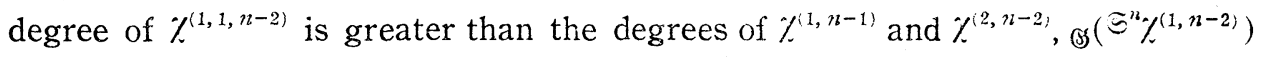
contains $\mathscr{S}^{(1,1, n-2)}$ only once. By lemma $5, \mathbb{S}$ is a transitive group and so we have ${ }^{\mathbb{B}}\left(\mathfrak{G}_{1} \mathcal{K}^{(1, n-2)}\right)={ }_{\mathbb{S}}\left(\mathbb{S}^{n} \chi^{(1, n-2)}\right)$. This is a contradiction.

Theorem 4. Let 1 bo a permutation group of degree $n \geqq 6$. If the character (S) $\%^{(1,2, n-3)}$ is irreducibie, then (B) is 5 -ply transitive. If the characters $\AA^{(1,1,1, n-3)}$ and ${ }^{5} \%^{(3, n-3)}$ are irreducible, then $\mathbb{S}$ is quadruply transitive, and moreover, if $n \geqq 8$ and contains an element $R$ such that $\alpha_{1}(R) \geqq 2$ and $\alpha_{3}(R) \geqq 1$, then $\mathbb{B}$ is 5-ply transitive.

Proof. Case I. We assume that of $\AA^{\{1,2, n-3)}$ is irreducible. Since, by G. Frobenius' formula, the degree of $\chi^{(1,2, n-3)}>$ the degrees of $\chi^{(1,1,1, n-3)}, \chi^{(3, n-3)}$, $\chi^{(2, n-2)}, \chi^{(1,1, n-2)}$ and $\chi^{(1, n-3)}$, we have $\Xi^{n}\left(\omega^{5} \varkappa^{(1,2, n-3)}\right)=\chi^{(1,2, n-3)}+$ a sum of irreducible characters whose dimensions are at least 4 , and so 


$$
\begin{aligned}
\Im^{n-1}\left(\Im^{n}\left(\mathfrak{S} \%^{(1,2, n-3)}\right)\right)= & \chi^{(2, n-3)}+\varkappa^{(1,1, n-3)}+\%^{(1,2, n-4)} \\
& + \text { a sum of irreducible characters whose } \\
& \text { dimensions are at least } 3 .
\end{aligned}
$$

On the other hand, since $B$ is transitive by Lemma 5 , this is equal to

$$
\mathbb{S}^{n-1}\left(\mathbb{S}_{1} \chi^{(1,2, n-3)}\right)=\mathbb{S}^{n-1}\left(\mathfrak{G}_{1} \chi^{(2, n-3)}\right)+\mathbb{S}^{n-1}\left(\mathfrak{G}_{1} \mathcal{Z}^{(1,1, n-3)}\right)+\mathbb{S}^{n-1}\left(\mathfrak{G}_{1} \chi^{(1,2, n-4)}\right)
$$

where $\mathscr{S}_{i}$ is the subgroup $\mathbb{B} \cap \mathfrak{S}_{1}^{n}$ of $\mathfrak{S}_{1}^{n}\left(=\mathfrak{S}^{n-1}\right)$ and $\chi^{(2, n-3)}, \chi^{(1,1, n-3)}, \ldots$ are irreducible characters of $\Im^{n-1}$. Hence $\mathscr{S}_{1} \mathcal{X}^{(2, n-3)}$ and $\mathscr{S}_{1} \chi^{(1,1, n-3)}$ are irreducible. From theorem $2 \mathbb{B}_{1}$ is quadruply transitive, and so $\mathbb{B}$ is 5 -ply transitive.

Case II. We assume that ${ }_{\infty} \chi^{(3, n-3)}$ and $\chi^{(1,1,1, n-3)}$ are irreducible. If (s) $\chi^{(1,2, n-3)}$ is irreducible, our assertion is contained in Theorem 2 , and so we may assume that of $\chi^{(1,2, n-3)}$ is reducible.

i) Proof of quadruple transitivity of 8 . Since the degree of $\chi^{(1,2, n-3)}>$ the degree of $\chi^{(1,1,1, n-3)}$ by Frobenius formula we have either

$$
\begin{aligned}
\mathscr{S}^{n}\left(\mathfrak{S}^{(1,1,1, n-3)}\right) & =\chi^{(1,1,1, n-3)} \\
& \left.=\chi^{(1,1,1, n-3)}+\mathcal{Z}^{(1,2, n-3)}\right\} \\
& +\mathrm{a} \text { sum of irreducible characters whose } \\
& \text { dimensions are at least } 4 .
\end{aligned}
$$

and so either

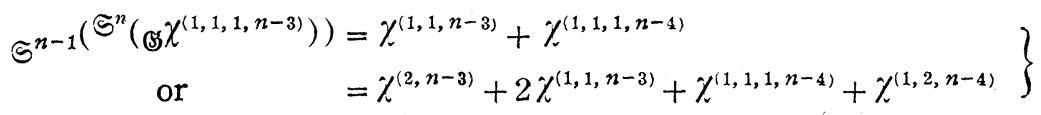

$$
\begin{aligned}
& + \text { a sum of irreducible characters whose }
\end{aligned}
$$

On the other hand, from the transitivity of $\&$ we have

$$
\begin{aligned}
& \mathbb{S}^{n-1}\left({ }^{n} \leftrightarrows\left(\mathcal{S}^{(1,1,1, n-3)}\right)\right)=\mathbb{S}^{n-1}\left(\mathbb{S}_{1} \chi^{(1,1,1, n-3)}\right) \\
& =\mathbb{S}^{n-1}\left(\mathfrak{S}_{1} \mathcal{K}^{(1,1, n-3)}\right)+\mathbb{S}^{n-1}\left(\mathfrak{S}_{1} \mathcal{K}^{(1,1,1, n-4)}\right) \text {. }
\end{aligned}
$$

If (7) is the case, it is easy to see, from (9), (11) and Theorem 3 , that $\mathbb{B}_{1}$ is triply transitive, and so $\&$ is quadruply transitive. Hence we assume that (8) is the case and in the similar way we may assume

$$
\begin{aligned}
\Im^{n}\left(\mathcal{S}^{(3, n-3)}\right)= & \mathcal{X}^{(3, n-3)}+\mathcal{\chi}^{(1,2, n-3)} \\
& + \text { a sum of irreducible characters whose } \\
& \text { dimensions are at least } 4 .
\end{aligned}
$$


From (8) and (12) we have

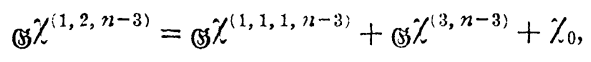

where $\%=1-3 \alpha_{3}$ by Frobenius' formula. By the comparison of the degrees of $\chi^{(1,2, n-3)}, \chi^{(1,1,1, n-3)}$ and $\chi^{(3, n-3)}$, we see that $\chi_{0}$ is a linear character, and so $\%$ is the identity character and $\alpha_{\ddot{s}}(R)=0$ for all elements $R$ of $\&$ and particularly

$$
3+\mid(3 \mid \text {. }
$$

From (10) and (11) ()$^{(1,1, n-3)}$ is either irreducible or a sum of exactly two irreducible characters $\psi_{1}$ and $\gamma_{2}$ of $\mathbb{B}$. From the transitivity of $\mathbb{B}$ we have

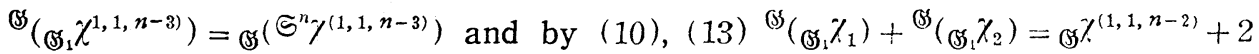
$\leftrightarrow \chi^{(1,1,1, n-3)}+\leftrightarrow \chi^{(3, n-3)}+1$. Hence an irreducible component of $\mathcal{S}^{(1,1, n-3)}$, say $\chi_{0}$, is the identity character, and so degree $\psi_{1}=$ degree $\chi^{(1,1, n-3)}-1=\frac{(n-2)(n-3)}{2}$ $-1=\frac{n^{2}-5 n+4}{2}$. Therefore $|\&|$ must be divisible by $\frac{n^{2}-5 n+4}{2}$. This contradicts with (14).

ii) Proof of 5-ple transitivity of $\mathbb{B}$. Since $\mathbb{B}$ is quadruply transitive, $\& \%^{(1,1, n-2)}$ and $\dot{\mathcal{K}}^{(2, n-2)}$ are irreducible by Theorem 2 and so, from $n \geqq 8$, si $\%^{(2, n-2)}$, (s) $\%^{(1,1, n-2)}$, (s) $\chi^{(3, n-3)}$ and ${ }^{(1)} \chi^{(1,1,1, n-3)}$ are distinct irreducible characters. Thus we have

$$
\begin{aligned}
0 & =\sum_{R \in \mathbb{S}}\left(\operatorname{SS}^{(1,1,1, n-3)}(R)+\mathbb{S} \chi^{(3, n-3)}(R)\right)\left(\mathbb{G} \chi^{(1,1, n-2)}(R)+\mathbb{S} \%^{(2, n-2)}(R)\right) \\
& =\frac{1}{3} \sum_{R \in \mathbb{S}} \alpha_{1}^{5}(R)+2 \sum_{R \in \mathbb{S}} \alpha_{1}^{2}(R) \alpha_{3}(R)+\sum_{R \in(S)} F\left(\alpha_{1}, \alpha_{3}\right)(R),
\end{aligned}
$$

where $F\left(\alpha_{1}, \alpha_{3}\right)$ is a class function whose weight is 4 . Since $\sum_{R \in \mathbb{S}}\left(\begin{array}{c}\alpha_{1}(R) \\ 5\end{array}\right)$ and $\sum_{R \in \mathscr{S}}\left({ }^{\alpha_{1}(R)}\right)\left(\alpha_{3}^{\left(\alpha_{3}(R)\right.}\right)$ are non zero, we have $\sum_{R \in(\mathcal{S}} \alpha_{1}^{5}(R)=\left(\tau(5)+u_{0}\right)|\mathbb{S}|$ and $\sum_{R \in \mathbb{S}} \alpha_{1}^{2}(R) \alpha_{3}(R)$ $=\left(\tau(2,0,1)+u_{1}\right)|\mathbb{S}|$ where $u_{0}$ and $u_{1}$ are non negative rational numbers from Lemma $3, \mathrm{ii})$ and $3^{\prime}$. Hence we have $\frac{\tau(5)+u_{0}}{3}+2 \tau(2,0,1)+2 u_{1}+\wedge_{0}=0$ where $\Lambda_{0}$ is the rational number determined by $F\left(\alpha_{1}, \alpha_{3}\right)$. On the other hand from

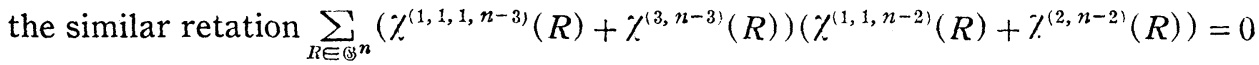
we have $\frac{\tau(5)}{3}+2 \tau(2,0,1)+\wedge_{0}=0$. Hence $s_{0}=1$ i.e. $\sum_{R \in \circlearrowleft} \alpha^{5}(R)=\tau(5)$. This proves the 5-ple transitivity of $(B$ by Lemma $3, i)$.

\section{§4. Remarks}

Theorem 3 in the last section is best possible in the sense that the irredu. 
cibility of one (and not two) of characters $\mathbb{S}^{(1,1, n-2)}$ and $\mathbb{S}^{(2, n-2)}$ of $\$ 3$ does not entail the quadruple transitivity of 0 . We can see this as follows:

i) The case of $7^{(2, n-2)}$ : The alternating group $\mathfrak{H}^{5}$ of degree 5 is exactly triply transitive, and it is well known that the character $9 l^{6 \chi^{(2,3)}}$ is irreducible. ${ }^{5)}$

ii) The case of $\chi^{(1,1, n-2)}$ : The Mathieu group $\mathfrak{M}_{22}$ of degree 22 whose order $g$ is $22 \cdot 21 \cdot 20 \cdot 48=443520$ is an example such that the irreducibility of $\sin ^{(1,1, n-2)}$ does not imply the quadruple transitivity of $\left(\mathfrak{S} . \mathfrak{M}_{22}\right.$ is defined as follows ${ }^{6}$ : Let $L F(3,4)$ be the special projective group consisting of all special projective transformations on 2-dimensional projective space $\mathscr{S}$ over $G F(4)$. Then we can easily see that $\operatorname{LF}(3,4)$ is a doubly transitive group on all (i.e. 21$)$ points $(x, y$, z) of $\mathscr{P}$ and the order of $\operatorname{LF}(3,4)$ is $21 \cdot 20 \cdot 48$. Then $\mathfrak{M}_{22}$ is the permutation group on 22 letters, $\{(x, y, z) \in \mathscr{P}\}$ and a new symbol I, generated by $\operatorname{LF}(3,4)$ and a permutation $S=\left(x, y, z \rightarrow x^{2}+y z, y^{2}, z^{2}\right) \cdot(1,0,0 \mid \mathrm{I})$. Evidently $\mathfrak{M}_{22}$ is exactly triply transitive. The subgroup $\mathscr{S}=\left\{\left(\begin{array}{lll}1 & 0 & \alpha \\ 0 & \gamma & \beta \\ 0 & 0 & \gamma^{-1}\end{array}\right) \mid \alpha, \beta, \gamma \in G F(4)\right.$ and $\left.\gamma \neq 0\right\}$ of $\mathfrak{M}_{22}$ consists of permutations which fix $(0,1,0),(1,0,0)$ and $I$, and $\Re=\left\{\left(\begin{array}{lll}1 & 0 & \alpha \\ 0 & 1 & \beta \\ 0 & 0 & 1\end{array}\right)\right\}$ $\alpha, \beta \in G F(4)\}$ and $\mathfrak{U}=\left\{\left(\begin{array}{ccc}1 & 0 & 0 \\ 0 & \gamma & 0 \\ 0 & 0 & \gamma^{-1}\end{array}\right) \mid \gamma \in G F(4)\right.$ and $\left.\gamma \neq 0\right\}$ are respectively 2 - and 3- Sylow subgroups of $\mathfrak{S}$, and they fix respectively 6 and 4 letters $\{(0,1,0)$, $(1,0,0), I,(1,1,0),(2,1,0),(3,1,0)\}$ and $\{(0,1,0),(1,0,0), I,(0,0,1)\}$ and types of elements in $\mathfrak{N}$ and $\mathfrak{U}$ are $(2)^{8}$ and $(3)^{6}$. Let $K_{i}$ be the subset of elements of $\mathfrak{M}_{22}$ fixing exactly $i$ letters and $\mathfrak{M}_{22}=K_{0}+K_{1}+\cdots+K_{22}$ and $K_{i} \cap K_{j}=\phi$ for $i \neq j$, and let $g_{i}$ be the number of elements in $K_{i}$. Then from the structure of $\mathfrak{M}_{22}$ it is easy to see that, except $K_{0}, K_{1}, K_{2}, K_{1}, K_{6}$ and $K_{22}, K_{i}$ 's are empty, and there exist elements of types $(11)^{2},(6)^{2},(3)^{3},(2)^{2}$ or $(8)^{2},(4),(2)$ in $K_{0}$, type $(7)^{3}$ in $K_{1}$, types $(5)^{4}$ or $(4)^{1}(2)^{2}$ in $K_{2}$, Type $(3)^{6}$ in $K_{4}$, type $(2)^{8}$ in $K_{6}$ and type $(1)^{22}$ in $K_{22}$. Further the order of the normalizer $\Re\left(\Re_{i}\right)$ of a 7 -Sylow group $\mathfrak{H}_{i}$ of $\mathfrak{M}_{22}$ (e.g. the cyclic subgroup generated by an element of type $(7)^{3}$ in $K_{1}$ ) is equal to the order of normalizer of a 7 -Sylow group of $L F(3,4)$, i.e.

5) See [2], [5] or [6].

6) See [7]. 
equal to $7 \cdot 3$, ${ }^{\prime \prime}$ and so, by the Sylow theorem, $g_{1}=\frac{443520}{7 \cdot 3} \times 6=126720$. Since $\mathfrak{M}_{22}$ is triply transitive, we have (by Lemma $3, i$ ))

$$
\begin{aligned}
g & =g_{0}+g_{1}+g_{2}+g_{4}+g_{6}+g_{22} \\
g & =g_{1}+2 g_{2}+4 g_{4}+6 g_{6}+22 g_{22} \\
2 g & =g_{1}+4 g_{2}+16 g_{4}+37 g_{6}+484 g_{22} \\
5 g & =g_{1}+8 g_{2}+64 g_{4}+216 g_{6}+10648 g_{22}
\end{aligned}
$$

Here $g=443520, g_{1}=126720$ and $g_{22}=1$, and so we have $g_{0}=173040, g_{2}=130284$, $g_{4}=12320$ and $g_{0}=1155$ from the above equations. Now we can culculate $\sum_{R \in \mathfrak{M}_{22}}\left\{\mathfrak{m}_{22} \chi^{(1,1,20)}(R)\right\}$ using $\chi^{(1,1,20)}=\frac{\left(\alpha_{1}-1\right)\left(\alpha_{1}-2\right)}{2}-\alpha_{2}$ and the above results, thus

$$
\begin{aligned}
\left.\sum_{R \in \mathbb{M}_{22}}\left\{\mathfrak{M}_{22}\right\}^{(1,1,20)}(R)\right\}^{2} & \left.=\sum_{i} \sum_{R \in K_{i}}\left\{\mathfrak{m}_{22}\right\}^{(2,20)}(R)\right\}^{2} \\
& =\left\{\chi^{(1,1,20)}(1)\right\}^{2}+1155\left\{\chi^{(1,1,20)}\left(R_{6}\right)\right\}^{2} \\
+ & 12320\left\{\chi^{(1,1,20)}\left(R_{4}\right)\right\}^{2}+A\left\{\chi^{(1,1,20)}\left(R_{2}\right)\right\}^{2}+B\left\{\chi^{(1,1,20)}\left(R_{0}\right)\right\}^{2}
\end{aligned}
$$

where $R_{i} \in K_{i}$ and $A<130284$ and $B<173040$, and so

$$
<210^{2}+1155 \times 2^{2}+12320 \times 3^{2}+130284 \times 2^{2}+173040 \times 1^{2}=853776<2 g .
$$

Therefore $\sum_{R \in \mathfrak{M}_{22}} \mathfrak{m}_{22} \chi^{(1,1, n-2)}(R)=g$. This means that the character is irreducible.

\section{REFERENCES}

[1] L. E. Dickson, Linear Groups, Leipzig (Teubner) 1901.

[2] G. Frobenius, Über die Charaktere der symmetrischen Gruppe, Sitzungsber. Preuss. Akad. (1900), pp. 516-534.

[3] — Über die Charaktere der alternierenden Gruppe, Sitzungsber. Preuss. Akad. (1901), pp. 303-315.

[4] - Über die Charaktere der mehrfach transitiven Gruppe, Sitzungsber. Preuss. Akad. (1904), pp. 558-571.

[5] D. E. Littlewood, The Theory of Group Characters, Oxford 1940.

[6] H. Weyl, The Classical Groups, Princeton 1938.

[7] E. Witt, Die 5-fach transitiven Gruppen von Mathieu, Abh. Math. Sem. 'Univ. Hamburg, Bd. 12 (1938), pp. 256-264.

\section{Mathematical Institute}

Nagoya University

7) See [1], § 238 . 